\title{
MODELLING OF TEXTILE COMPOSITE REINFORCEMENTS ON THE MICRO-SCALE
}

\author{
Oliver Döbrich*, Thomas Gereke, Chokri Cherif
}

Institute of Textile Machinery and High Performance Material Technology, Technische Universität Dresden, Hohe Straße 6, 01069 Dresden, Germany

Email: oliver.doebrich@tu-dresden.de

\begin{abstract}
:
Numerical simulation tools are increasingly used for developing novel composites and composite reinforcements. The aim of this paper is the application of digital elements for the simulation of the mechanical behaviour of textile reinforcement structures by means of a finite element analysis. The beneficial computational cost of these elements makes them applicable for the use in large models with a solution on near micro-scale. The representation of multifilament yarn models by a large number of element-chains is highly suitable for the analysis of structural and geometrical effects. In this paper, a unit cell generating method for technical reinforcement textiles, using digital elements for the discretization, is introduced.
\end{abstract}

\section{Keywords:}

digital elements, finite element simulation, textile reinforcements, unit cell

\section{Introduction}

The development of novel textile composite reinforcements changes from excessive production and analysis processes to an all-virtual development chain. The textile product can be regarded in dependence of the yarn construction and the process parameters. The resulting mechanical values can be analysed by virtual tests and optimisation steps can be carried out on a virtual level. The trend of virtual engineering of textile products is due to reduction of costs and development time for new products. For the structural and mechanical analysis of textiles, mostly representative unit cells (RUCs) are used. The periodic construction of most textiles allows the analysis of the structure and the mechanical behaviour on these small units. The geometry of the textile structure mainly depends on the used yarn material and the production parameters. Virtual methods enable examination of the structure in dependence of the production parameters and examination of the mechanical behaviour in dependence of the textile structure without manufacturing the actual textile product. This can be considered as the main advantage in the development chain of textile products. In the last decade, numerical drape simulations have increasingly been used to predict the textile's ability to form into a complex three-dimensional shape [1]. Here, the textile mechanical behaviour is used as input data for the simulations and also as fundamental for comparing different textile materials. This is only one field of application that shows the excessive use of textile mechanical parameters. For performing virtual tests or other simulations on these RUCs, the correct cell geometry has to be known. The specific geometry of fabrics can be obtained from mathematical approaches or experimental tests, like microscopic or microtome analyses. Accurate results depend on the geometric description, correct boundary conditions and the yarn model, which is mostly a continuum approach [2]. The yarn material used, the yarnto-yarn friction and the textile bonding structure cause the resulting fabric rigidities and their non-linearities. Classic RUCs are traditionally built up by mathematical approaches. Initial penetrations are avoided for a robust simulation. The constructed RUCs based on mathematical assumptions can be seen rather as approximations than as realistic images of the unit cell geometry. Due to the multi-scale nature of textile structures, simulations can be carried out on different levels of objectivity. Depending on the structural level, textiles can be regarded as a continuum material on the macro-level [3]. Textiles can also be analysed on the yarn level, which is denoted as the meso-level. Here, yarns and their geometric bound are mostly described explicitly with a continuum approach for the yarn mechanics. As most yarns in technical textiles are multifilament yarns and made of thousands of filaments, the simulation could also be carried out on the micro-level. Here, simulation techniques are required that allow a discretization of a technical multifilament yarn into this large number of filaments. A representation of each filament by solid elements, and additionally with more than one element representing the cross-section, is very intensive due to the computational costs. Such examinations are only reasonable when there is the need for analysing the cross-sectional deformation of the filaments, as done by Nilakantan [4]. Otherwise, simplifications of the yarn mechanics can be made. The digital element approach was introduced for textile simulation purposes [5]. This approach will be used in this paper for a near micro-scale resolution of the used multifilament yarns. The use of digital elements is very suitable for examination of cross-sectional deformations [6], which will be analysed here.

This paper deals with the construction of several RUCs composed of digital elements. These elements allow a near micro-scale resolution of technical highperformance multifilament yarns, which makes them predestined for structural analysis. The introduced method for the unit cell generation was already introduced by Döbrich and colleagues 
[7] for a continual multifilament yarn model. The process of virtually generating the geometry of different fabrics will be performed in dependence of their production parameters to analyse the resulting structure and geometry in dependence of these parameters. These investigations enable examination of the structural geometry and mechanical properties in a complete virtual methodology. The results of the virtual generation process were compared with the real textile structure of the examined fabrics. The result of this novel method was a unit cell model with an excellent initial yarnto-yarn contact behaviour, accurate frictional effects and initial pre-stresses as well as complexly deformed cross-sections of the embedded multifilament yarns. The introduced technique was able to deliver RUC geometries from textiles, which were excellent for predicting the textile's mechanical behaviour by virtual mechanical tests. In this paper, this method gets further improved and used to generate unit cells with multifilament yarn models discretized with digital elements to achieve a near micro-scale resolution of the structure.

\section{Materials}

The examination for this paper was done for a plain woven fabric and leno woven fabrics. These two different textile constructions mainly differ in the kind of the yarn construction. Whereas the yarns in the plain woven fabric cross each other at every point of intersection, the yarns in the leno woven fabric are straight aligned but bound together by a binding warp yarn. Both examined textiles were composed of technical E-glass multifilament yarns. The characteristics of the used materials are presented in Tables 1 and 2.

Table 1. Characteristics of examined plain woven fabric

\begin{tabular}{|c|c|c|c|}
\hline & \multicolumn{2}{|c|}{ Material properties } & Microscope and scanning images \\
\hline $\begin{array}{l}\text { Yarn material: } \\
900 \text { tex EC-GF }\end{array}$ & $\begin{array}{l}\text { Yarn height weft } \\
\text { Yarn width weft: } \\
\text { Yarn height warp } \\
\text { Yarn width warp } \\
\text { Cross-sectional area weft } \\
\text { Cross-sectional area warp }\end{array}$ & $\begin{array}{l}0.2675 \mathrm{~mm} \\
3.1715 \mathrm{~mm} \\
0.3016 \mathrm{~mm} \\
2.8258 \mathrm{~mm} \\
2.6653 \mathrm{~mm}^{2} \\
2.6775 \mathrm{~mm}^{2}\end{array}$ & 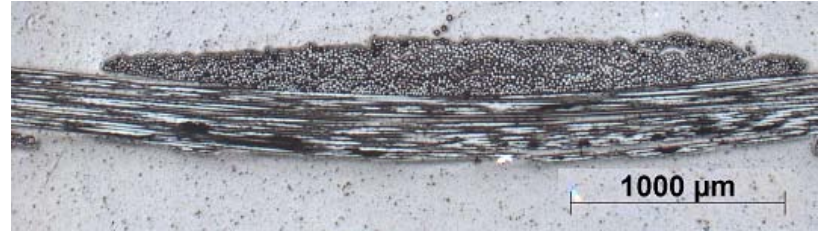 \\
\hline $\begin{array}{c}\text { Fabric material: } \\
\text { Plain woven } \\
\text { fabric }\end{array}$ & $\begin{array}{l}\text { Fabric thickness } \\
\text { Crimp of weft yarns } \\
\text { Crimp of warp yarns } \\
\text { Weft distance } \\
\text { Warp distance }\end{array}$ & $\begin{array}{l}0.5517 \mathrm{~mm} \\
0.3454 \% \\
0.17285 \% \\
\sim 3.33 \mathrm{~mm} \\
\sim 3.63 \mathrm{~mm}\end{array}$ & \\
\hline
\end{tabular}

Table 2. Characteristics of examined leno woven fabrics

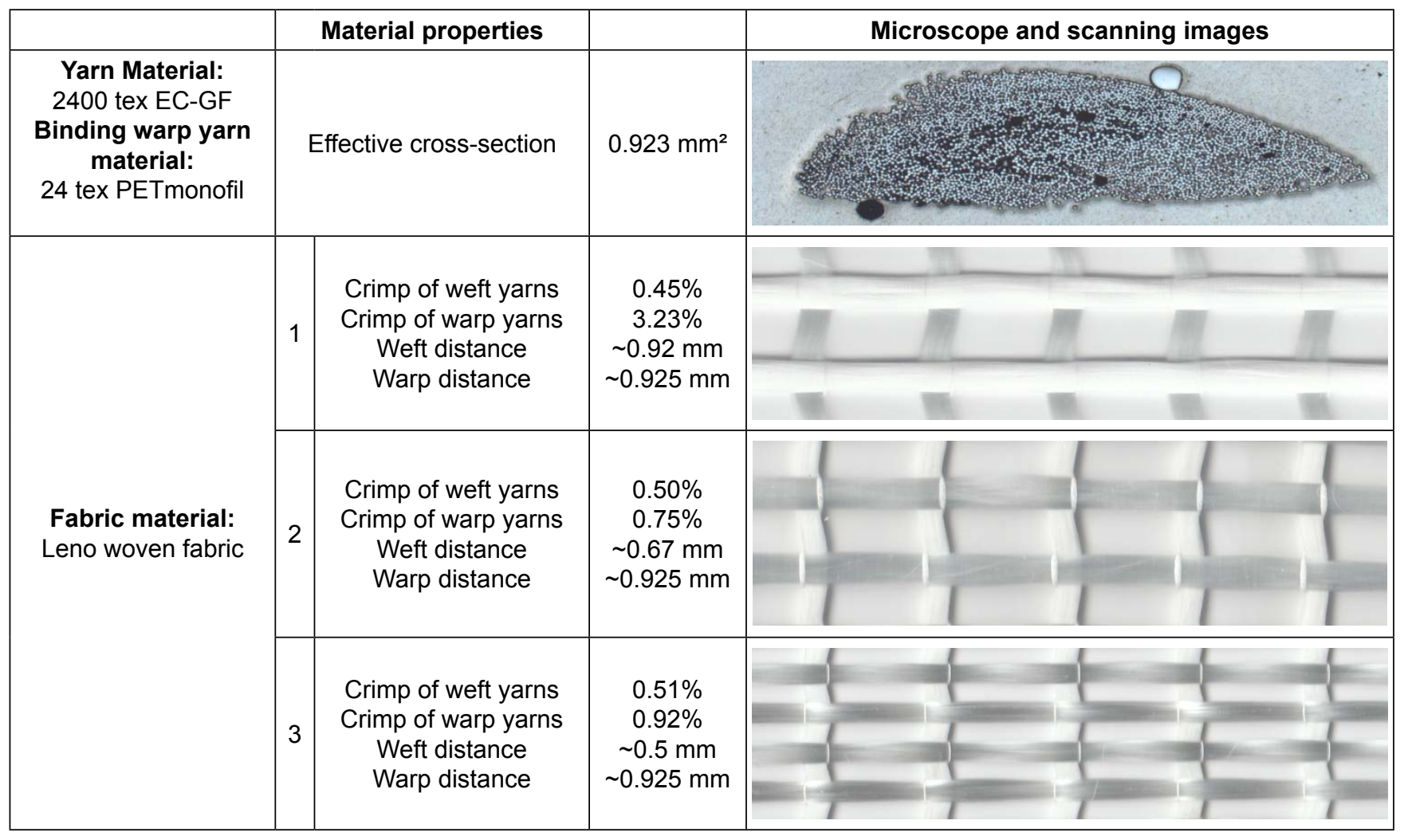


Examination concerning the accurateness of the introduced method is done by comparing the actual shape of the yarns' cross section at different points with the solution of the generating process, which is explained in Section 5. A quantitative observation will be done by comparing the warp and weft yarn trace of the actual manufactured samples with the computed trace of the introduced method.

\section{Techniques for generating unit cells from the state of the art}

\subsection{Mathematical approach}

One of the mainly used approaches for setting up the structural dimensions of a woven unit cell is the mathematical approach. Here, the traces of the yarns are determined by mathematical functions. Possibly the most referenced literature regarding this topic is from McBride and Chen [8]. They introduced equations for the description of the yarn trace in a plain woven fabric from geometrical relationships. These equations are commonly used for a geometrical description of a plain woven unit cell [9].

\subsection{Microscopic and microtome optical analyses}

Another more extensive approach for gaining information about the yarn alignments in a unit cell is by performing optical analysis on the examined materials. These approaches have the benefit that the dimensions and the geometry are reproduced directly from the actual textile, which makes it very accurate. The disadvantage is that there is the need for a sample of the examined material. The method of a microtome sectioning analysis was described by Rief et al. [10]. Slices of a resin embedded material were cut from the sample and two-dimensional images were taken from every slice. Image processing reproduced the three-dimensional structure of the examined material [10]. Another possibility is to examine the woven geometry by microscopic analysis, as Hivet and Boisse introduced [11]. The conditions are equal to the microtome analysis, which is explained before. Some important concerns are stated in the paper. It is known that the injection of a dry fabric with resin influences the observed sample. As seen from the resulting images, resin infiltrates the fabric and the filaments of the multi-filament yarn. Therefore, the results of a microtome or a microscopic measurement are not identical as for a dry fabric. This disadvantage is known but accepted for most examinations.

\subsection{Generating unit cells of textile structures}

Some other techniques for generating unit cell geometries, or at least substitution models, were introduced in the past. Tan et al. [12] introduced a method to compound a woven unit cell out of micro- and macro-blocks [12]. Each block has another mechanical property, according to its position in the unit cell. A combination of these blocks results in a substitution continuum for predicting the mechanical values of fabrics. Wang et al. [13] presented an approach comparable with the one introduced in the current paper. Here, digital elements were also used. A non-continuum approach was realised, which enabled a "near filament-level resolution" [13]. An initial geometry was built by pre-processing. The final woven geometry was obtained by a relaxation process, where the yarn cross-sections and the trace of the yarns adapt to achieve a static equilibrium. By applying a pre-tensile load to the yarns an initial impulse is generated to change the yarn geometry. The obtained geometry contains compressed yarns due to contact. The contact forces are due to the pretension. It is possible that the amount of forces used for pretensioning the initial configuration has only a negligible effect on the resulting geometry. However, the resulting contact forces are not realistic due to the freely chosen value for the pre-tensile forces.

\section{The digital element approach}

Digital elements are simplified beam elements, which are pinjointed to build an element-chain. They were firstly referenced by Wang and Sun in 2001 [5]. This simulation technique was already introduced for textile structure simulations. Digital elements are characterised by a reduced stiffness matrix, $K$, as:

$$
K=\frac{E \cdot A}{\Delta L}\left[\begin{array}{cccccc}
1 & 0 & 0 & -1 & 0 & 0 \\
0 & 0 & 0 & 0 & 0 & 0 \\
0 & 0 & 0 & 0 & 0 & 0 \\
-1 & 0 & 0 & 1 & 0 & 0 \\
0 & 0 & 0 & 0 & 0 & 0 \\
0 & 0 & 0 & 0 & 0 & 0
\end{array}\right]=\left[\begin{array}{cccccc}
G & 0 & 0 & -G & 0 & 0 \\
0 & 0 & 0 & 0 & 0 & 0 \\
0 & 0 & 0 & 0 & 0 & 0 \\
-G & 0 & 0 & G & 0 & 0 \\
0 & 0 & 0 & 0 & 0 & 0 \\
0 & 0 & 0 & 0 & 0 & 0
\end{array}\right],
$$

where $E$ is the Young's modulus and $A$ is the cross-sectional area of an element-chain. According to Wang and Sun [5], the values of the occupied terms $G$ are not to be regarded as the classical stiffness matrix terms. Every term has to be "a large positive value" [5]. Additional terms are added to the matrix to prevent singularity. Another assumption is the pin-jointed connection between the elements. The bending stiffness of a single filament is negligible. Therefore, a pin-joint assumption is a consequent simplification. The digital elements are not as cost extensive as normal beam elements, which has benefits in large models with a multitude of interacting filaments. Zhou et al. [6] introduced multi-chain digital elements in 2003. These chains are bundles of single filament models to represent multifilament yarns. These yarns consist of hundreds of single filaments. Discretizing a single yarn into these hundreds of single filaments is unusual due to the large amount of interactions, the enormous pre-processing effort and, thus, the high computational costs. Miao et al. [14] examined the amount of digital chains that is necessary for a multifilament yarn discretization. Miao et al. showed that there is no need in discretizing a multifilament yarn with the exact number of existing filaments. The number of element-chains rather has to be merely large enough to provide "sufficient accuracy" [14]. For braiding simulations, satisfactory results were achieved with the usage of 19 chains per yarn. A weaving simulation delivered accurate results by using 50-70 chains per yarn [14]. Thus, the multi-chain digital element models are beneficial for modelling multifilament yarns on a level of objectivity that is "near to filament" [14]. 


\section{Novel method for generating unit cells on a near microscale solution}

As mentioned before, this method was already introduced by the author with a continual approach for the multifilament yarn mechanics [7]. The aim of the introduced method is to achieve a yarn configuration that equates the one of the examined textile in its static equilibrium. In this paper, the behaviour of the digital elements is analysed regarding this objective. The benefit of the introduced method, rather than modelling the geometry from input data, is that the yarn is straight and stressless in the beginning of the simulation. Here, no strains are applied. Afterwards, the aligned yarn segments are forced to come up with the textiles' yarn construction by prescribed motions. Preliminary tests showed that the sequence of prescribed motions is secondary as long as the boundaries are correct; at least as the yarns' material model is purely elastic as it is for the examination in this study. Firstly, all geometric dimensions of the examined textiles have to be known. These parameters are the warp and weft density, the yarn crimp in every direction and the yarn dimension. Figure 1a) shows the initial alignment of the yarns before the unit cell construction begins. All contacts are disabled at this state because of the yarn penetrations. The unit cell borders are defined and correspond to the warp and weft densities previously determined by optical analysis. The borders are fixed and represent the boundaries for the symmetric conditions. The crimp of the yarns is also considered. After aligning the yarns, the boundaries of prescribed motion are disabled and reduced to a minimum required for realising the symmetry conditions. Figure 1 describes this approach demonstratively for the generation of a plain woven unit cell.

\section{Results}

As a result of the introduced method, accurate models of textile unit cells are achieved. The resulting unit cells consist of yarns that are under pre-strain and therefore under initial pre-stress. This enables the consideration of initial friction, which can be seen as a big advantage of this method. For the validation of this technique, the achieved models were compared with images of the actual textile. The traces of the yarns as well as the shapes of the multifilament yarn cross-sections were compared. Figure 2 shows the assessment of the plain woven unit cell model.

As an additional benefit, the introduced method enables examination of the textile structure in terms of the input parameters needed for performing the generating method. For example, by performing the same generation process with a varied parameter for the crimp, analyses in dependence of the yarn tension while manufacturing the structure can be performed. This is due to the fact that the structure achieved by this method results from a static equilibrium that takes the yarn stresses into account. Figure 3 shows the comparison of three different leno woven unit cell structures, as manufactured. In Figure 4a), one of this unit cell models is shown separately and Figure $4 \mathrm{~b}$ ) shows the predicted change in the structure by using less yarn tension for the warp yarn and binding warp yarn while production. As a result of this, the crimp of the warp yarn increases.

\section{Conclusions}

Digital elements are suitable for modelling technical multifilament yarns. Textile models composed out of digital

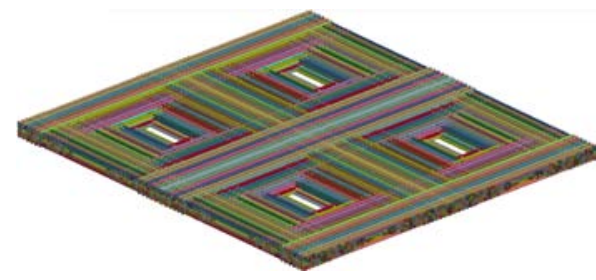

a) Initial configuration

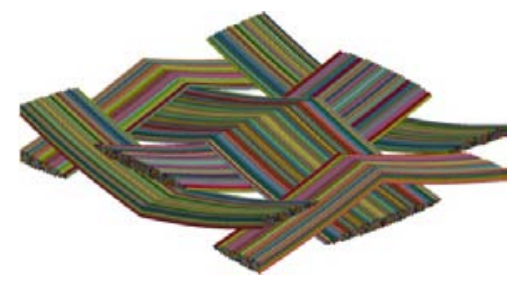

b) Prescribed configuration

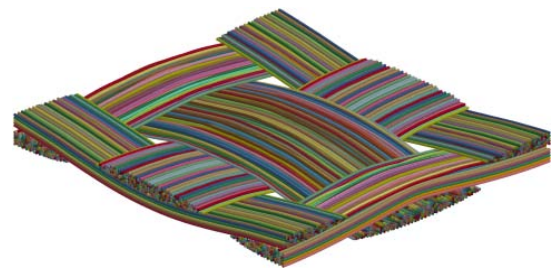

c) Static equilibrium

Figure 1 Steps of plain woven fabric unit cell generation process

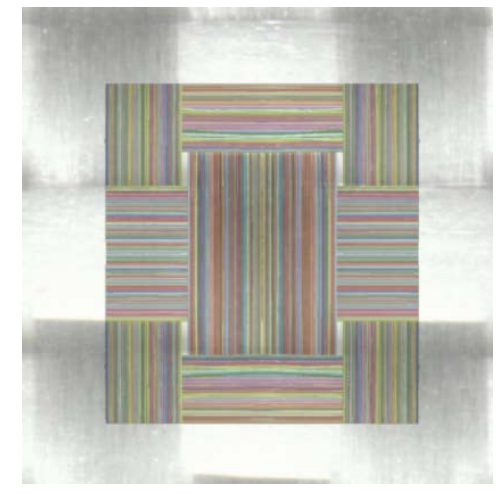

Comparison of the plain woven fabric model with the actual plain woven fabric
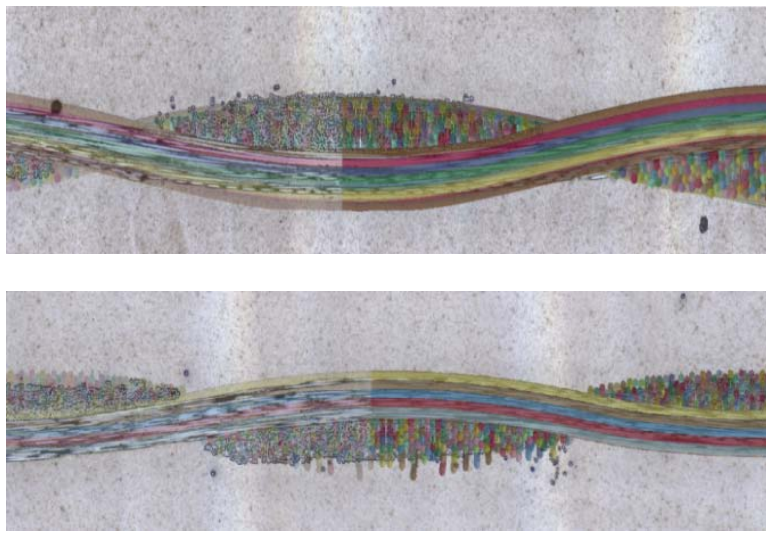

Comparison of the simulated cross-section and the yarn trace with microscopic images

Figure 2 Comparison of the results from unit cell generation with the actual plain woven fabric 


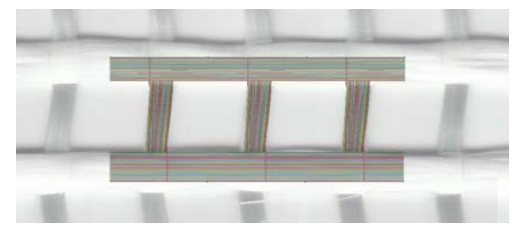

Leno woven structure \#1

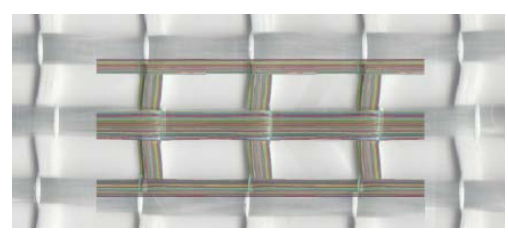

Leno woven structure \#2

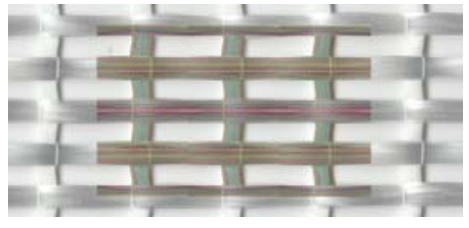

Leno woven structure \#3

Figure 3 Comparison of different models generated for leno woven structures
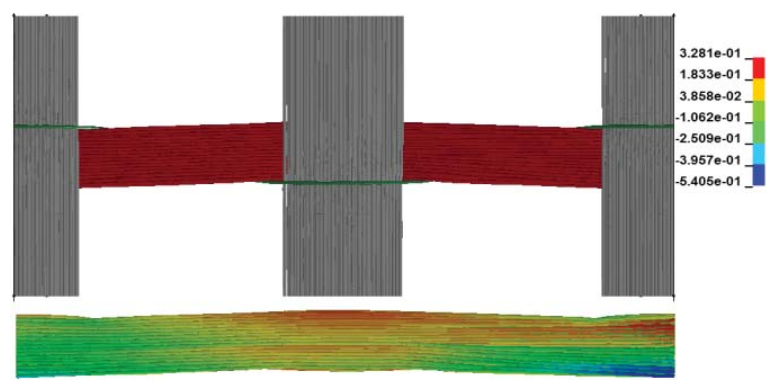

a) Unit cell model of leno woven structure

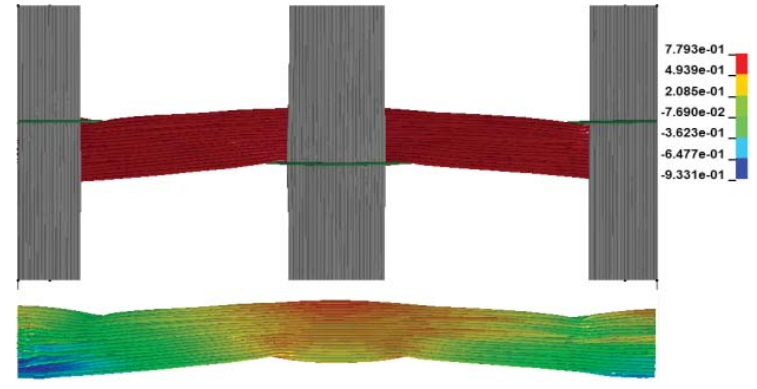

b) Structural change of the leno woven by changing production parameters

Figure 4 Analysis of the leno woven structure in function of the process parameters (scale shows out-of-line displacement of warp yarn)

multi-element chains are useful for analysing textiles' geometrical structure. The influence of different stress states of the yarns while producing the textiles can be examined. The analysis of the mechanical behaviour with the use of the digital elements is still outstanding.

This paper has been previously published at the $4^{\text {th }}$ ITMC Lille Metropole 2013 International Conference in Roubaix, France (9-11 October 2013).

\section{Acknowledgement}

Financial support for this work by the European Social Fund

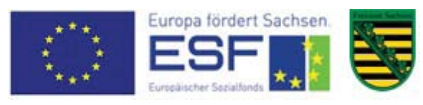
and the Free State of Saxony is gratefully acknowledged.

\section{References}

[1] Gereke, T.; Döbrich, O.; Hübner, M.; Cherif, C.: Experimental and computational composite textile reinforcement forming: A review, Composites Part A: Applied Science and Manufacturing, Vol. 46, pp. 1-10, ISSN 1359-835X, 2013.

[2] Grujicic, M.; Arakere, G.; He, T.; Gogulapati, M.; Cheeseman, B. A.:A numerical investigation of the influence of yarn-level finite-element model on energy absorption by a flexible-fabric armour during ballistic impact, Proceedings of the Institution of Mechanical Engineers, Part L: Journal of Materials Design and Applications, Vol. 222, pp. 259276, 2008

[3] Döbrich, O.; Gereke, T.; Diestel, O.; Krzywinski, S.; Cherif, C.: Decoupling the bending behavior and the membrane properties of finite shell elements for a correct description of the mechanical behavior of textiles with a laminate formulation, Journal of Industrial Textiles, doi: 10.1177/1528083713477442, online first.
[4] Nilakantan, G.: Filament-level modeling of Kevlar KM2 yarns for ballistic impact studies, Composite Structures, Vol. 104, pp. 1-13, ISSN 0263-8223, 2013

[5] Wang, Y.; Sun, X.: Digital-element simulation of textile processes, Composites Science and Technology, Vol. 61(2), pp. 311-319, ISSN 0266-3538, 2001.

[6] Guangming Zhou, Xuekun Sun, Youqi Wang, Multi-chain digital element analysis in textile mechanics, Composites Science and Technology, Vol. 64(2), pp. 239-244, ISSN 0266-3538, 2004.

[7] Döbrich, O.; Gereke, T.; Cherif, C.: Virtual Weaving - Construction of a woven representative unitcell, Proceedings of 13th AUTEX World Textile Conference 2013, 22-24 May, Dresden, Germany

[8] McBride, T. M.; Chen, J.: Unit-cell geometry in plain-weave fabrics during shear deformations, Composites Science and Technolgy, Vol. 57(3), pp. 345-351, ISSN 0266-3538, 1997.

[9] Aitharaju, V. R.; Averill, R. C.: Three-dimensional properties of woven-fabric composites, Composites Science and Technology, Vol. 59(12), pp. 1901-1911, ISSN 0266-3538, 1999.

[10] Rief, S.; Glatt, E.; Laourine, E.; Aibibu, D.; Cherif, C.; Wiegemann, A.: Modelling and CFD-simulation of woven textiles to determine permeability and retention properties, AUTEX Research Journal, Vol. 11(3), pp. 78-83, 2011.

[11] Hivet, G.; Boisse, P.: Consistent $3 D$ geometrical model of fabric elementary cell. Application to a meshing preprocessor for $3 D$ finite element analysis. Finite Elements in Analysis and Design, Vol. 42(1), pp. 25-49, 2005.

[12] Tan, P.; Tong, L.; Steven, G. P.: A three-dimensional modeling technique for predicting the linear elastic property of opened-packing woven fabric unit cells, Composite Structures, 38(1997)1-4, pp. 261-271, ISSN 0263-8223 
[13] Wang, Y.; Miao, Y.; Swenson, D.; Cheeseman, B. A.; Yen, C.-F.; LaMattina, B.: Digital element approach for simulating impact and penetration of textiles, International Journal of Impact Engineering, Vol. 37(5), pp. 552-560, 2010.
[14] Miao, Y.; Zhou, E.; Wang, Y.; Cheeseman, B. A.: Mechanics of textile composites: Micro-geometry, Composites Science and Technology, Vol. 68(7-8), pp. 1671-1678, ISSN 02663538, 2008. 